\title{
Tracking Mobile Users in Wireless Networks via Semi-Supervised Co-Localization
}

\author{
Jeffrey Junfeng Pan, Sinno Jialin Pan, Jie Yin, Lionel M. Ni Fellow, IEEE and Qiang Yang Fellow, IEEE
}

\begin{abstract}
Recent years have witnessed growing popularity of sensor and sensor-network technologies, supporting important practical applications. One of the fundamental issues is how to accurately locate a user with few labelled data in a wireless sensor network, where a major difficulty arises from the need to label large quantities of user location data, which in turn requires knowledge about the locations of signal transmitters, or access points. To solve this problem, we have developed a novel machine-learning-based approach that combines collaborative filtering with graph-based semi-supervised learning to learn both mobile-users' locations and the locations of access points. Our framework exploits both labelled and unlabelled data from mobile devices and access points. In our two-phase solution, we first build a manifold-based model from a batch of labelled and unlabelled data in an offline training phase and then use a weighted k-nearest-neighbor method to localize a mobile client in an online localization phase. We extend the two-phase co-localization to an online and incremental model that can deal with labelled and unlabelled data that come sequentially and adapt to environmental changes. Finally, we embed an action model to the framework such that additional kinds of sensor signals can be utilized to further boost the performance of mobile tracking. Compared to other state-of-the-art systems, our framework has been shown to be more accurate while requiring less calibration effort in our experiments performed at three different test-beds.
\end{abstract}

Index Terms-Wireless sensor networks, Semi-supervised learning, Indoor localization, Co-localization, Al applications.

\section{INTRODUCTION}

Locating users in a wireless network is an important task in many applications that range from context-aware computing [1], location-based services [2], [3] to robotics [4], [5]. With recent advances in pervasive computing and mobile technology, the problem of tracking wireless devices using received-signal-strength (RSS) has attracted intense interest in many research communities [6], [7]. RSS-based tracking or localization is a challenging task since radio signals usually attenuate in a highly nonlinear and uncertain way in a complex environment where client devices may be moving. Existing approaches to RSS-based localization fall into two main categories: (1) radio propagation models [8]; (2) statistical machine learning models [9], [10], [11]

Traditionally, practitioners have used geometric models that are based on signal propagation properties and access point locations. These models have poor accuracy when the access points (APs) are separated far from each other as in cellphone base towers. More recent works have used learning-based models that can achieve much better accuracy. These learning based models are set up purely from the client devices based on a large amount of calibration data [9], [10], [11].However, a major problem with the learning-based models is that, in many indoor localization cases, the calibrated training data are

- Jeffrey J. Pan is with Facebook Inc., Palo Alto, CA, USA (e-mail: panjunfeng@gmail.com)

- Sinno J. Pan is with the Institute for Infocomm Research, 1 Fusionopolis Way, \#21-01 Connexis, Singapore 138632 (email:sinnocat@gmail.com).

- Jie Yin is with the Information Engineering Laboratory, CSIRO ICT Centre, Australia (e-mail:Jie.Yin@csiro.au).

- Lionel M. Ni and Qiang Yang are with the Department of Computer Science and Engineering, the Hong Kong University of Science and Technology, Hong Kong, China (e-mail: \{ni,qyang\}@cse.ust.hk). manually collected since the Global Positioning System (GPS) may not work in an indoor environment. The data collection process is time consuming, and can be easily outdated, making it necessary for us to collect the data over and over again. In order to reduce the calibration effort, this work attempts to answer the following three questions:

- How can we reduce calibration effort to build a tracking system by incorporating unlabelled data?

- Can we further enhance the performance if the locations of some access points are known?

- Can we make use of different kinds of signals to further boost the performance?

In this paper, we address the problem of simultaneously recovering the locations of both mobile devices and access points, which we call co-localization, using labelled and unlabelled RSS data from both mobile devices and access points. We propose two solutions to this problem. The first one is called two-phase co-localization which is based on semisupervised manifold-learning techniques, which has an offline training phase and an online localization phase. However, a two-phase model may not adapt to environmental changes well since the model remains unchanged after being trained. To solve this problem, we extend the model to online colocalization which can cope with calibrated and uncalibrated data stream in real-time and adjust itself online.

\section{- Solution I: Two-Phase Co-Localization}

In general, learning-based systems using RSS values function in two phases : an offline training phase and an online localization phase. In the offline phase, a learning-based model is trained by using the signal strength values received from the access points at selected locations in the area of interest. These values comprise the training data gathered from 
a physical region, which are used to calibrate a probabilistic location-estimation system. In the online localization phase, the real-time signal strength samples received from the access points are used to estimate the current location based on the learned model.

More specifically, in the offline training phase, we take two steps for model building. In the first step, we assume that only unlabelled RSS data are given. We show that the problem can be solved by Latent Semantic Indexing (LSI) or Singular Value Decomposition (SVD) [12] techniques that are popular in information retrieval. Consequently, the relative locations of access points and mobile device trajectories can be determined. In the second step, we assume that a small amount of labelled RSS data from mobile devices and access points are given. To recover the absolute locations of the devices and access points, we apply a semi-supervised algorithm with graph Laplacian and manifold learning [13], [14]. Finally, we provide a unified framework for both the above unsupervised and semi-supervised solutions. A preliminary version of this solution can be found in [15].

\section{- Solution II: Online Co-Localization}

However, in many applications, access points can not be deployed in a static environment where calibrated and uncalibrated data arrive in a streaming manner. Access points may be removed, relocated and added for better coverage and link quality. In each case, a localization system may gradually become inaccurate without costly re-calibration and re-running the whole training process. It is also wasteful to discard previous computational results even if the system can be retrained. A better idea is to construct an online localization model in a streaming manner.

The online co-localization extends the two-phase framework and addresses the problem of recovering the locations of both mobile devices and access points from radio signals that come in a streaming manner, by exploiting both labelled and unlabelled data from mobile devices and access points. The solution is based on online and incremental manifold-learning techniques [16], [17] and semi-supervised techniques [14] that can cope with labelled and unlabelled data that come sequentially. A preliminary version of online co-localization can be found in [18].

\section{- Extension: Sensor Fusion with Action Models}

Note that the above two solutions rely on measuring signal strength values sent from static landmarks such as wireless access points to mobile devices. Localization systems can also be broadly classified into two categories: Landmark-based and Landmark-free, depending on what sensor devices are used. Landmark-based systems rely on a certain proximity measurement between a mobile device and multiple landmarks that are deployed in the environment [19], [20]. Typical landmarks can be satellites in GPS or access points in WiFi Networks. In an indoor environment, satellite signals are not always available. Instead, WiFi access points are deployed in many buildings. However, accurate tracking mobile devices using RSS is a challenging task since RSS values have large noise in a complex indoor environment due to attenuation, shadowing and multi-path effects.

Landmark-free systems can perform self-localization with- out relying on any external references [21]. For example, a mobile robot can locate itself because an action sequence is usually available. The robot can update its status after executing an action such as move(forward, 1 meter) or turn $\left(\right.$ left, $\left.90^{\circ}\right)$, which means the robot is "to move forward 1 meter" or "to turn left 90 degrees", respectively. Similarly, an Inertial Navigation System (INS) has motion sensors such as gyroscope, accelerometer and compass, which can be used for inferring the action of a mobile user such as speed and orientation, walking or not, etc. Landmark-free systems can be very accurate for a short time. However, errors may be accumulated due to sensor noise if no landmarks are available for re-calibration.

Hence, a better idea is to combine the Landmark-based and Landmark-free systems. In this paper, we extend the proposed co-localization solutions by utilizing both signal strength received from landmarks and readings from motion sensors. Specifically, we use the action sequences inferred from compass and accelerometer, and reconstruct the location trajectory via semi-supervised manifold learning techniques. We borrow and extend the idea from [22] in the sense that if action $_{i}$ and action $_{j}$ are similar, the change of status or location would be similar. Our method is called Localization via Action Respecting Manifold (LARM).

\section{Related Works}

In the past, propagation models were widely used for location estimation due to their simplicity and efficiency [23]. These models usually assume that access points are labelled, e.g., their locations are known. An alternative is to apply machine learning methods to learn a model that captures the correlations between RSS values and locations [7]. With these methods the location information of access points need not to be known. Instead, they usually rely on models that are trained with RSS data collected on a mobile device and the corresponding labels or physical locations [9], [24], [11]The training data are usually collected offline. These signal values may be noisy and nonlinear due to environmental dynamics. Therefore, sufficient data have to be collected to power algorithms for approximating the signal to location mapping functions using histograms [24], k-nearest-neighbors (KNN) [9], etc.

Besides semi-supervised learning models, transfer learning techniques have been also applied to the RSS-based localization problem to reduce the calibration effort [7]. The goal of transfer learning is to learn a precise model in a target domain with as few as training data by making use of training data from a related domain, where the data distribution may be different from that of the target domain [25].

However, these transfer-learning-based models only focused on tracking the mobile device, while our proposed colocalization framework can recover the locations of access points and track the mobile device simultaneously. By assuming an action model be given, Ferris et al. [19] proposed an unsupervised framework for SLAM (simultaneous localization and mapping) [5] in a WiFi environment. It has been observed in [22] that two identical actions lead to similar status change. 
By treating actions as discrete labels, latent coordinates can be recovered via Action Respecting Embedding [22].

\section{Methodology}

\subsection{Problem Statement}

Consider a two-dimensional co-localization problem ${ }^{1}$. Assume that a user holds a mobile device and navigates in an indoor wireless environment $\mathcal{C} \subseteq \mathbb{R}^{2}$ with $n$ access points, which can periodically send out beacon signals. At some time $t_{i}$, the RSS values from all the $n$ access points are measured by the mobile device to form a row vector $\mathbf{s}_{i}=\left[\begin{array}{llll}s_{i_{1}} & s_{i_{2}} & \ldots & s_{i_{n}}\end{array}\right] \in \mathbb{R}^{n}$. A sequence of $m$ signal strength vectors form an $m \times n$ matrix $S=\left[\begin{array}{llll}\mathbf{s}_{1}^{\prime} & \mathbf{s}_{2}^{\prime} & \ldots & \mathbf{s}_{m}^{\prime}\end{array}\right]^{\prime}$, where $\mathbf{s}_{i}^{\prime}$ denotes a transposition of $\mathbf{s}_{i}$. Here, the locations of some access points and the mobile devices at some time $t_{i}$ are known or labelled, while the rest are unlabelled.

We estimate the $m \times 2$ location matrix $P=$ $\left[\mathbf{p}_{1}^{\prime}, \mathbf{p}_{2}^{\prime}, \ldots, \mathbf{p}_{m}^{\prime}\right]^{\prime}$ where $\mathbf{p}_{i}=\left[\begin{array}{ll}p_{i_{1}} & p_{i_{2}}\end{array}\right] \in \mathcal{C}$ is the location of the mobile device at time $t_{i}$ and the $n \times 2$ location matrix $Q=\left[\mathbf{q}_{1}^{\prime}, \mathbf{q}_{2}^{\prime}, \ldots, \mathbf{q}_{n}^{\prime}\right]^{\prime}$ where $\mathbf{q}_{j}=\left[q_{j_{1}} q_{j_{2}}\right] \in \mathcal{C}$ is the location of the $j^{\text {th }}$ access point. We call this problem co-localization. More specifically, we have two main objectives:

- Two-phase co-localization. Given a fixed amount of labelled and unlabelled data collected offline, the first objective is to build a model for simultaneously recovering the locations of the remaining unknown access points and the trajectory of the mobile device. The model can then be used for online localization. The model remains unchanged in the online phase unless we re-train everything. These offline and online phases are done in a way as most traditional machine learning approaches do.

- Online co-localization. Assuming that partially calibrated data come sequentially, the second objective is to determine and update the locations of the remaining unlabelled access points and the trajectory of the mobile device in real-time. Note that $m$ is not a constant value. As time elapses, $m$ may increase from $1,2, \ldots$, to any number. We wish to dynamically adjust the model when observing new data without relying on an offline training phase.

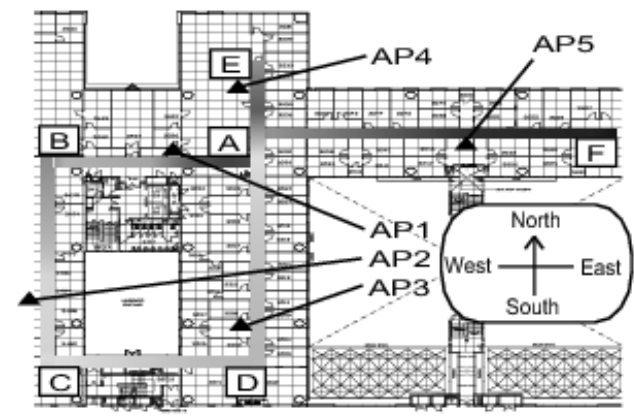

Fig. 1. An indoor WLAN Test-bed.

Example 1 As an example, Figure 1 shows an indoor 802.11 wireless LAN environment of size about $60 \mathrm{~m} \times 50 \mathrm{~m}$, which

1. Note that it is straight-forward to extend our proposed models to threedimensional co-localization problems. has $n=5$ access points. A user with an IBM T42 notebook that is equipped with an Intel Pro/2200BG internal wireless card walks from $A$ through $B, C, D, A, E$ to $F$ at time $t_{A}, t_{B}, t_{C}, t_{D}, t_{A^{\prime}}, t_{E}, t_{F}$. Correspondingly, a total number of $m=1,2, \ldots, 7$ signal strength vectors are incrementally extracted. The final $7 \times 5$ matrix $S$ is shown in Table 1 . By walking from $A$ to $F$ in the hallways, we collected 500 signal strength vectors from 5 access points. Note that the blank cells denote the missing values, which we can fill in a small default value, e.g., $-100 \mathrm{dBm}$.

TABLE 1

Signal Strength (unit:dBm).

\begin{tabular}{|c|c|c|c|c|c|}
\hline & $A P_{1}$ & $A P_{2}$ & $A P_{3}$ & $A P_{4}$ & $A P_{5}$ \\
\hline$t_{A}$ & -40 & & -60 & -40 & -70 \\
\hline$t_{B}$ & -50 & -60 & & -80 & \\
\hline$t_{C}$ & & -40 & -70 & & \\
\hline$t_{D}$ & -80 & & $\mathbf{- 4 0}$ & -70 & \\
\hline$t_{A^{\prime}}$ & -40 & & -70 & -40 & -60 \\
\hline$t_{E}$ & -40 & & -70 & -40 & -80 \\
\hline$t_{F}$ & -80 & & & -80 & -50 \\
\hline \multicolumn{7}{|c|}{ (All values are rounded for illustration) }
\end{tabular}

Our first task is to estimate the trajectory matrix $P$ of the mobile device at all times and to determine the location matrix $Q$ of the access points $A P_{1}, A P_{2}, \ldots, A P_{5}$. Our second task is to dynamically update the trajectory matrix $P$ of the mobile device at each time when new data come and to update the location matrix $Q$ of the access points in an online manner.

\subsection{Domain Characteristics}

There are four main characteristics about RSS by observing the data in Table 1:

1) Considering two rows of the data, the mobile device at two different time may be spatially close if their pairwise signal strengths are similar from most access points, e.g., the time $t_{A}$ and $t_{A^{\prime}}$.

2) Considering two columns of the data, two access points may be spatially close if their pairwise signal strength values are similar most of the time, e.g., $A P_{1}$ and $A P_{4}$.

3) Considering a single cell $s_{i j}$ of the data, the mobile device and the $j$ access point may be spatially close to each other at time $t_{i}$ if the signal is strong, e.g., the mobile device is close to $A P_{3}$ at time $t_{D}$.

4) Considering two neighbored rows of the data, the mobile device at two consecutive time may be spatially close if their time interval is small by assuming that a user may not move too fast or too irregularly. For example, the locations of the mobile device at time $t_{A^{\prime}}$ and $t_{E}$ are close since $\left|t_{A^{\prime}}-t_{E}\right|<\Delta T$.

\subsection{SVD-based Relative Co-Localization}

Given unlabelled data only, we can determine the relative locations of the mobile device and the access points. Not surprisingly, the relative co-localization is closely related to Latent Semantic Indexing (LSI) [12]. In this view, we treat an access point as a term and a mobile device at some time as a document. The first three observed characteristics mentioned 
above would be mapped to the similarities of documentdocument, term-term and document-term respectively. Estimating the positions of the mobile device and the access points corresponds to discovering the latent semantics of documents and terms in some concept space.

More specifically, we can estimate the relative coordinates by performing Singular Value Decomposition (SVD).

1) Transform the signal matrix $S=\left[s_{i j}\right]_{m \times n}$ to a nonnegative weight matrix $\widetilde{S}=\left[\widetilde{s}_{i j}\right]_{m \times n}$ by a linear function $\widetilde{s}_{i j}=s_{i j}-s^{m i n}$, where $s^{m i n}$ is the minimal signal strength detected, e.g., the noise level or $-100 \mathrm{dBm}$.

2) Normalize the weight matrix by $\widetilde{S}_{\mathcal{N}}=D_{1}^{-1 / 2} \widetilde{S} D_{2}^{-1 / 2}$. Here, $D_{1}$ and $D_{2}$ are both diagonal matrices such that $D_{1}=\operatorname{diag}\left(d_{1}^{1}, d_{2}^{1}, \ldots, d_{m}^{1}\right)$ where $d_{i}^{1}=\sum_{j=1}^{n} \widetilde{s}_{i j}$ and $D_{2}=\operatorname{diag}\left(d_{1}^{2}, d_{2}^{2}, \ldots, d_{n}^{2}\right)$ where $d_{j}^{2}=\sum_{i=1}^{m} \widetilde{s}_{i j}$.

3) Perform SVD on the normalized weight matrix by $\widetilde{S}_{\mathcal{N}} \approx$ $U_{m \times r} \Sigma_{r \times r} V_{n \times r}^{\prime}$. The columns of $U_{m \times r}=\left[\mathbf{u}_{1} \ldots \mathbf{u}_{r}\right]$ and $V_{n \times r}=\left[\mathbf{v}_{1} \ldots \mathbf{v}_{r}\right]$ are the left and right singular vectors. The singular values of the diagonal matrix $\Sigma_{r \times r}=$ $\operatorname{diag}\left(\sigma_{1}, \sigma_{2}, \ldots, \sigma_{r}\right)$ are ranked in non-increasing order.

4) The (latent) location matrices of the mobile device $P$ and that of the access points $Q$ can be estimated using $P=D_{1}^{-1 / 2}\left[\begin{array}{ll}\mathbf{u}_{2} & \mathbf{u}_{3}\end{array}\right]$ and $Q=D_{2}^{-1 / 2}\left[\begin{array}{ll}\mathbf{v}_{2} & \mathbf{v}_{3}\end{array}\right]$. Note that we skip the first singular vectors $\mathbf{u}_{1}$ and $\mathbf{v}_{1}$ which mostly capture some constant since matrix $\widetilde{S}_{\mathcal{N}}$ is not centering.

As an example, after performing SVD on data in Example 1, we obtained the latent coordinates of the mobile device and the access points, which are shown in Figure 2(a). In this example, it is easy to see that the hallway structure is not well preserved by comparing the true location sequence shown in Figure 1. This is because SVD assumes a linear subspace, while the correlation of RSS values and distance to access points is often nonlinear [11].

A better solution is using kernelized SVD [26], by transforming signal strength values to weights by a nonlinear function. More specifically, we transform the signal matrix $S=\left[s_{i j}\right]_{m \times n}$ to a new weight matrix $\widetilde{S}=\left[\widetilde{s}_{i j}\right]_{m \times n}$ by a Gaussian function:

$$
\widetilde{s}_{i j}=\exp \left(-\left|s_{i j}-s^{\max }\right|^{2} / 2 \sigma^{2}\right)
$$

where $s^{\max }$ is the maximal signal strength detected, e.g., the signal strength around an access point, and $\sigma$ is a parameter of the Gaussian kernel, which is known as kernel width. Figure 2(b) plots the co-localization result using $P$ and $Q$. Intuitively, the reconstructed hallway structure and the locations of access points are better than that shown in Figure 2(a) while referring to the ground truth illustrated in Figure 1.

\subsection{Manifold-based Absolute Co-Localization}

When the physical locations of some access points and the mobile device at some time are known, we can ground the unknown coordinates by exploiting the geometry of the signal distribution. More specifically, we can use manifold-based learning, which generally assumes that if two points are close in the intrinsic geometry of the marginal distribution, their conditional distributions are similar [27]. This implies that mobile devices would be spatially close to each other if their signal vectors are similar along some manifold structure [28]. For example, the mobile device at time $t_{A}$ and $t_{E}$ would be spatially close to each other (Figure 1) since their signal strength values are similar (Table 1).

A more concrete example is shown in Figure 3. As can be seen in Figure 3(a), there is a two-dimensional triangle localization area with three beacon nodes placed at the vertices. The corresponding signal strength values form a twodimensional nonlinear signal manifold in a three-dimensional space in Figure 3(b). Point $A, B$ and $C$ are neighbors in both location and signal spaces.

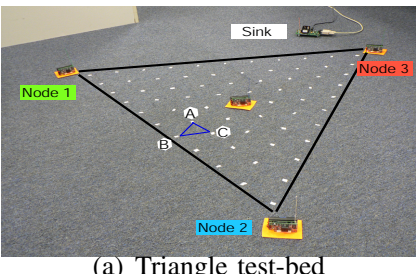

(a) Triangle test-bed

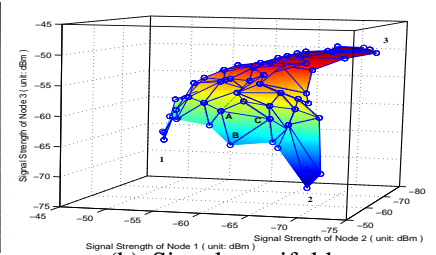

(b) Signal manifold

\section{Fig. 3. Neighborhood preserving.}

When the manifold assumption holds, the optimal solution is given by $\mathbf{f}^{*}=\arg \min \Sigma_{i=1}^{l}\left|f_{i}-y_{i}\right|^{2}+\gamma \mathbf{f}^{T} L \mathbf{f}$ [14], where the first term measures the fitting error and the second term poses the smoothness along the manifold and $L$ is the graph Laplacian [29]. For our problem, the objective is to optimize:

$$
P^{*}=\underset{P \in \mathbb{R}^{m \times 2}}{\arg \min }\left(P-Y_{P}\right)^{\prime} J_{P}\left(P-Y_{P}\right)+\gamma_{P} P^{\prime} L_{P} P,
$$

where $P$ is the coordinate matrix of the mobile device to be determined; $J_{P}=\operatorname{diag}\left(\delta_{1}, \delta_{2}, \ldots, \delta_{m}\right)$ is an indication matrix where $\delta_{i}=1$ if the coordinate of the mobile device at time $t_{i}$ is given and otherwise $\delta_{i}=0 ; Y_{P}=\left[\mathbf{y}_{1}^{\prime}, \mathbf{y}_{2}^{\prime}, \ldots, \mathbf{y}_{m}^{\prime}\right]^{\prime}$ is an $m \times 2$ matrix supplying the calibration data where $\mathbf{y}_{i}$ is the given coordinate of the mobile device at time $t_{i}$ if $\delta_{i}=1$ and otherwise the value of $\mathbf{y}_{i}$ can be any, e.g., $\mathbf{y}_{i}=\left[\begin{array}{ll}0 & 0\end{array}\right] ; \gamma_{P}$ controls the smoothness of the coordinates along the manifold; $L_{P}=D_{P}-W_{P}$ is the graph Laplacian; $W_{P}=\left[w_{i j}\right]_{m \times m}$ is the weight matrix and $w_{i j}=\exp \left(-\left\|\mathbf{s}_{i}-\mathbf{s}_{j}\right\|^{2} / 2 \sigma^{2}\right)$ if $\mathbf{s}_{i}$ and $\mathbf{s}_{j}$ are neighbors along the manifold and otherwise $w_{i j}=0$; $D_{P}=\operatorname{diag}\left(d_{1}, d_{2}, \ldots, d_{m}\right)$ and $d_{i}=\sum_{j=1}^{m} w_{i j}$.

By setting the derivative of the right hand side in (2) to zero, we obtain the optimal solution shown as follows,

$$
P^{*}=\left(J_{P}+\gamma_{P} L_{P}\right)^{-1} J_{P} Y_{P} \text {. }
$$

Similarly, the coordinates of the access points can be obtained by solving the following optimization problem $Q^{*}=$ $\underset{Q \in \mathbb{R}^{n \times 2}}{\arg \min }\left(Q-Y_{Q}\right)^{\prime} J_{Q}\left(Q-Y_{Q}\right)+\gamma_{Q} Q^{\prime} L_{Q} Q$, and thus

$$
Q^{*}=\left(J_{Q}+\gamma_{Q} L_{Q}\right)^{-1} J_{Q} Y_{Q},
$$

where $L_{Q}=D_{Q}-W_{Q}$ is the graph Laplacian, $W_{Q}$ is the weight matrix and $D_{Q}$ is constructed from $W_{Q}$.

Thus, when the locations of the mobile device and the access points are partially known, we can co-localize them by solving Equations (3) and (4) respectively. Alternatively, we can combine them into a single equation as

$$
R^{*}=\left(J+\gamma_{B} L_{B}+\gamma_{C} L_{C}\right)^{-1} J Y,
$$




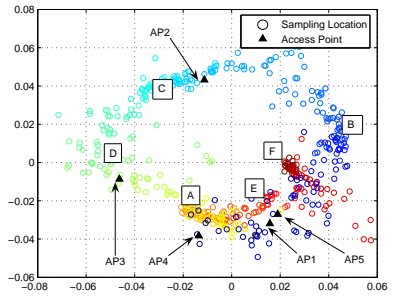

(a) (Linear) SVD co-localization

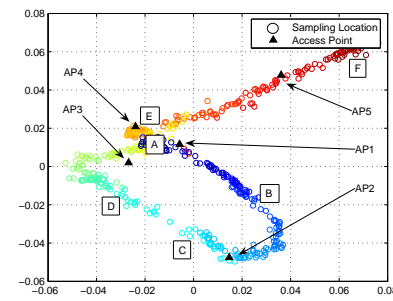

(b) (Nonlinear) SVD co-localization

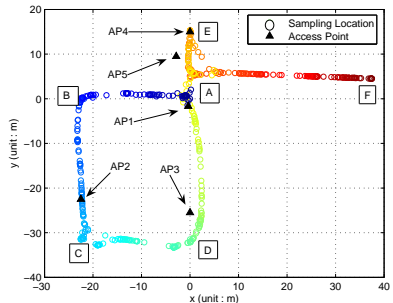

(c) Manifold-based co-localization

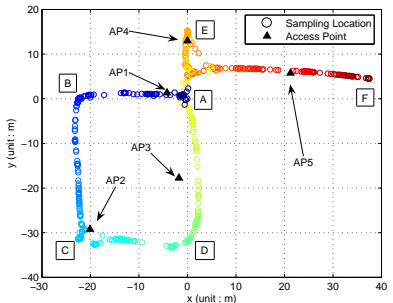

(d) The unifying framework

Fig. 2. 802.11 Wireless LAN test in an indoor environment.

where $R=\left[\begin{array}{ll}P^{\prime} & Q^{\prime}\end{array}\right]^{\prime}$ is the coordinate matrix of the mobile device and the access points; $Y=\left[\begin{array}{ll}Y_{P}^{\prime} & Y_{Q}^{\prime}\end{array}\right]^{\prime}$ gives the label information; $J=\left[\begin{array}{cc}J_{P} & 0 \\ 0 & J_{Q}\end{array}\right]$ is the indication matrix; $L_{B}=\left[\begin{array}{cc}L_{P} & 0 \\ 0 & 0\end{array}\right]$ and $L_{C}=\left[\begin{array}{cc}0 & 0 \\ 0 & L_{Q}\end{array}\right]$ are the graph Laplacians.

In practice, the graph Laplacians $L_{B}$ and $L_{C}$ in Equation (5) are normalized [13], [30]. Figure 2(c) shows an example of the manifold-based co-localization when the locations of the mobile device at time $t_{A}, t_{B}, t_{C}, t_{D}, t_{E}, t_{F}$ and the access points $A P_{2}, A P_{3}, A P_{4}$ are known. As can be seen, the trajectory of the mobile device is well grounded when compared to the ground truth shown in Figure 1. However, locations of access points are estimated badly, e.g., the location of $A P_{5}$. The reason is that in manifold-based co-localization, there are two manifolds, one is for WiFi data and the other is for access points. These two manifolds are learned separately. Most manifold-based methods require dense unlabelled data to propagate label information through an underlying manifold structure. However, from access points' perspective, the data are extremely sparse. Furthermore, $A P_{5}$ is far away from the other four. In this case, the manifold-based co-localization approach is not able to estimate the location of $A P_{5}$ accurately. In contrast, the SVD-based co-localization approach employs matrix factorization techniques to recover latent locations of the access points and WiFi data jointly. As a result, a lot of unlabeled WiFi data can help recover latent locations of the access points. Although the latent coordinates can not be aligned to absolute locations without label information, the relative distance between access points is more accurate than that estimated by the manifold-based co-localization approach. In the following, we propose to combine SVD-based and manifold-based co-localization to align the mobile device and the access points to the ground truth jointly.

\subsection{Solution I: Two-Phase Co-Localization}

Offline Training Phase So far, we have formulated the unsupervised co-localization based on SVD and the semisupervised co-localization based on the manifold assumption using Equation (5) by exploiting the correlation between the mobile device and the access points. In this section, we integrate them through a unifying framework. Essentially, performing SVD on $S_{\mathcal{N}}$ is equivalent to solving the following generalized eigenvalue problem [31]

$$
L_{A} Z=D_{A} Z \Lambda,
$$

where $L_{A}=D_{A}-W_{A}$ is the graph Laplacian, $W_{A}=\left[\begin{array}{cc}0 & \tilde{s} \\ \tilde{s}^{\prime} & 0\end{array}\right]$ and $D_{A}=\left[\begin{array}{cc}D_{1} & 0 \\ 0 & D_{2}\end{array}\right]$. The eigenvalues of the diagonal matrix $\Lambda=\operatorname{diag}\left(\lambda_{1}, \lambda_{2}, \ldots, \lambda_{m+n}\right)$ are ranked in non-decreasing order. $Z=\left[\mathbf{z}_{1}, \mathbf{z}_{2}, \ldots \mathbf{z}_{m+n}\right]$ are the eigenvectors. $\left[P^{\prime} Q^{\prime}\right]^{\prime}=$ $\left[\begin{array}{ll}\mathbf{z}_{2} & \mathbf{z}_{3}\end{array}\right]$. Note that we skip the first eigenvector $\mathbf{z}_{1}$ since the solution is trivial. Furthermore, it is interesting to see that we have $\lambda_{i}=1-\sigma_{i}$ where $i=1,2, \ldots, r$ [31]. Detailed analysis and comparison of LSI, SVD and graph Laplacian can be found in literatures on LSI [12], Bipartite Co-Clustering [31] and Fiedler Embeddings [32].

Putting (5) and (6) together, we aim to optimize:

$$
R^{*}=\underset{R \in \mathbb{R}^{(m+n) \times 2}}{\arg \min }(R-Y)^{\prime} J(R-Y)+\gamma R^{\prime} L R .
$$

The first term measures the fitting error and the second term constrains the smoothness among the mobile device and the access points. The solution is given by:

$$
R^{*}=(J+\gamma L)^{-1} J Y,
$$

where $L=\gamma_{A} L_{A}+\gamma_{B} L_{B}+\gamma_{C} L_{C}=D-W$.

We set $\gamma$ to a small positive value, which is directly related to harmonic functions on the graph such that the coordinate of a mobile device or an access point $\mathbf{r}_{i}$ in $R=\left[\mathbf{r}_{1}^{\prime}, \mathbf{r}_{2}^{\prime}, \ldots, \mathbf{r}_{m+n}^{\prime}\right]^{\prime}$ is determined by the average of its neighbors: $\mathbf{r}_{i}=\frac{\sum_{j} w_{i j} \mathbf{r}_{j}}{\sum_{j} w_{i j}}$, where $W=\left[w_{i j}\right]_{(m+n) \times(m+n)}=$ $\left[\begin{array}{cc}\gamma_{B} W_{P} & \gamma_{A} \widetilde{S} \\ \gamma_{A} \widetilde{S}^{\prime} & \gamma_{C} W_{Q}\end{array}\right]$.

In practice, we optimize the objective function over the normalized graph Laplacian [13], [30] to balance the weights of vertices by substituting $R=D^{-1 / 2} F$ into (7)

$$
F^{*}=\underset{F \in \mathbb{R}^{(m+n) \times 2}}{\arg \min }\left(D^{-1 / 2} F-Y\right)^{\prime} J\left(D^{-1 / 2} F-Y\right)+\gamma_{\mathcal{N}} F^{\prime} L_{\mathcal{N}} F,
$$

where $L_{\mathcal{N}}=D^{-1 / 2} L D^{-1 / 2}$ is the normalized graph Laplacian. The optimal $F$ is given by

$$
F^{*}=\left(J D^{-1 / 2}+\gamma_{\mathcal{N}} L_{\mathcal{N}}\right)^{-1} J Y .
$$

Substituting $F=D^{1 / 2} R$ back to Equation (10), the locations of the mobile device and the access points are given by

$$
R^{*}=D^{-1 / 2}\left(J D^{-1 / 2}+\gamma_{\mathcal{N}} L_{\mathcal{N}}\right)^{-1} J Y .
$$

We can export the estimated coordinates of the mobile device trajectory $P^{*}$ and the access point locations $Q^{*}$ from

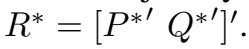


Online Localization Phase The location of a new signal strength vector $\mathbf{s}_{i}$ is predicted as follows:

1) Find the $k$ neighbors closest to $\mathbf{s}_{i}$ in the training data $S=\left[\begin{array}{llll}\mathbf{s}_{1}^{\prime} & \mathbf{s}_{2}^{\prime} & \ldots & \mathbf{s}_{m}^{\prime}\end{array}\right]^{\prime}$. Let $\mathcal{C}_{i}$ be the index set of the $k$ nearest neighbors. Besides, we link $\mathbf{s}_{i}$ to those access points from which we can detect the radio signal. We also link $\mathbf{s}_{i}$ to $\mathbf{s}_{i-1}$ in order to pose the temporal constraint by assuming that a user may not move too fast $\left(t_{i}-t_{i-1}<\Delta T\right)$. Denote the index set for these additional links as $\mathcal{B}_{i}$.

2) Approximately, we can predict the location using harmonic functions [33], which are smooth functions on the graph such that $\mathbf{r}_{i}$ is determined by the weighted average of its neighbors. This property holds if there is no uncertainty in the labelled locations of matrix $P$ during training $(\gamma \rightarrow 0$ in (7)),

$$
\tilde{\mathbf{r}}_{i} \approx \frac{\sum_{j \in \mathcal{C}_{i} \cup \mathcal{B}_{i}} w_{i j} \mathbf{r}_{j}}{\sum_{j \in \mathcal{C}_{i} \cup \mathcal{B}_{i}} w_{i j}} .
$$

Note that the above $\tilde{\mathbf{r}}_{i}$ is an approximation because adding $\mathbf{s}_{i}$ to the existing neighborhood graph from the training data may slightly change the graph structure. We link the $i^{\text {th }}$ node to the node set $\mathcal{C}_{i}$ but do not eliminate any existing edge in the graph to maintain the $k$-neighbor relationship among all nodes.

\subsection{Solution II: Online Co-Localization}

We will extend the above Two-Phase Co-Localization model to an online version. We wish that it can dynamically adjust itself when new data come sequentially in real-time. The key point is how to add the new data into the learned graph by updating the $k$-neighbor relationship and the corresponding weight matrix $W$. This can be done repeatedly in two online steps: Predict and Update.

Predict Given a new signal vector $\mathbf{s}_{i}$ at time $t_{i}$, we find its $k$ nearest neighbors and use Equation (12) in the above online localization phase for predicting the location $\tilde{\mathbf{r}}_{i}$.

Update The addition and deletion of nodes can modify the neighborhood graph and the corresponding graph Laplacian. We use the method described in [16] for updating the neighborhood graph structure locally.

- Node Addition Let $\mathcal{A}_{i}^{+}$and $\mathcal{D}_{i}^{+}$be the set of edges to be added and deleted after inserting $v_{i}$ to the neighborhood graph, respectively. Let $\tau_{j}$ be the index of the $k^{t h}$ nearest neighbor of $v_{j}$. Here we assume that all $\mathrm{k}$ nearest neighbors of $v_{j}$ have been ranked in non-decreasing order in terms of the distance to $v_{j}$. Given a $k$-nearest-neighborhood graph consisting of $n$ nodes, when the $(n+1)^{t h}$ node $v_{i}$ is inserted to the graph, we need to add $k$ edges to connect $v_{i}$ to its $k$ nearest neighbors, $e\left(v_{i}, v_{j}\right)$, where $v_{j} \in \mathcal{C}_{i}$. Furthermore, for each $v_{j}$ in the old graph, if $\Delta_{j, \tau_{j}} \leq \Delta_{j, i}$, where $\Delta_{j, i}$ denotes the distance between $v_{i}$ and $v_{j}$, then the $k$ nearest neighborhood of $v_{j}$ remains the same, thus the corresponding local neighborhood graph of $v_{j}$ does not need to be updated. Otherwise, if $\Delta_{j, \tau_{j}}>\Delta_{j, i}$, then $v_{i}$ replaces $v_{\tau_{j}}$ in the $k$ nearest neighborhood of $v_{j}$. Thus the corresponding neighborhood graph needs to be updated as follows:

$\mathcal{A}_{i}^{+}=\left\{e(j, i): j \in \mathcal{C}_{i}\right.$ or $\left.\Delta_{j, \tau_{j}}>\Delta_{j, i}\right\}$,

$\mathcal{D}_{i}^{+}=\left\{e\left(j, \tau_{j}\right): \Delta_{j, \tau_{j}}>\Delta_{j, i} \& \Delta_{\tau_{j}, j}>\Delta_{\tau_{j}, l_{j}}\right\}$,

where $l_{j}$ is the index of the $k^{\text {th }}$ nearest neighbor of

$v_{\tau_{j}}$ after inserting $v_{i}$ in the graph.

- Node Deletion Similarly, let $\mathcal{A}_{i}^{-}$and $\mathcal{D}_{i}^{-}$denote the set of edges to be added and deleted after removing $v_{i}$ from the neighborhood graph, respectively. The graph update can be done as follows:

$\mathcal{A}_{i}^{-}=\left\{e\left(i, h_{i}\right)\right\}$, where $h_{i}$ is the $(k+1)^{t h}$ nearest neighbor before removing $v_{i}$ in the graph.

$\mathcal{D}_{i}^{-}=\left\{e(i, j): j \in \mathcal{C}_{i}\right\}$.

After updating the neighborhood graph, it is straightforward to modify the corresponding weight matrix $W$. For an added edge $e(i, j)$, we set both the values of $w_{i j}$ and $w_{j i}$ because the neighborhood graph is symmetric. If it is a deleted edge, we clear the values of $w_{i j}$ and $w_{j i}$. The graph Laplacian $L=D-W$ can be updated in a similar way.

Finally, we have to re-estimate the location matrix $R=$ $\left[\begin{array}{ll}P^{\prime} & Q^{\prime}\end{array}\right]^{\prime}$ of the mobile devices and the access points so that it can reflect the change of the neighborhood graph and the new graph Laplacian $L$. Instead of using Equation (8) for solving $R$, we update $R$ by iteration. In each iteration cycle, we apply $\mathbf{r}_{i}^{\text {new }}=\frac{\sum_{j \in \mathcal{C}_{i} \cup \mathcal{B}_{i}} w_{i j} \mathbf{r}_{j}^{\text {old }}}{\sum_{j \in \mathcal{C}_{i} \cup \mathcal{B}_{i}} w_{i j}}, i=1, \ldots, m+n$. We use the predicted $\tilde{\mathbf{r}}_{i}$ as the initial values for iteration. Furthermore, the weight matrix $W$ does vary too much after addition or deletion. We can thus obtain very good estimation after a few iterations.

Example 2 A user with a mobile device walks in the office area shown in Figure 1. The mobile device periodically collects signal vectors. The user can mark down his location when he walks by some landmark points such as corners and dead-ends of the hallways $(A, B, \ldots, F)$. Thus, the data that come in a streaming manner are partially labelled. By applying the online co-localization method, we continuously update the recovered locations of the mobile devices and the access points. Figure 4 shows the online co-localizaiton results at six key frames when the user walks by $A, B, \ldots, F$. As can be seen, the locations of the user trajectory and the access points are dynamically calibrated when obtaining new data. For example, $A P_{3}$ gradually converges to its true location.

\subsection{Special Cases of Co-Localization}

Co-localization is a general framework for RSS-based tracking and mapping. It addresses the problem of simultaneously recovering the locations of both mobile devices and access points by exploiting both labelled and unlabelled data from mobile devices and access points. The model can be applied with or without an offline training phase. It is flexible since we can calibrate the system in many different ways, depending on what information we have at hand. For example, if a wireless provider is unable to provide us with some access point locations, we can still set up an accurate tracking system by collecting data ourselves. If the access point locations are partially known, we can use them and further enhance the 
performance. Some special cases of our model are summarized as follows:

- When only unlabelled RSS data collected by mobile devices and no location information of access points are available, we can do unsupervised dimension reduction and recover the relative coordinates of both access points and mobile devices as shown in Figure 2(b). It is related to a Gaussian Process Latent Variable Model to recover latent coordinates of user trajectories based on unlabelled data [19].

- When labelled RSS data collected by mobile devices and no location information of access points are available, the model acts similarly to a classical KNN-based method [9], which is applied for indoor tracking using WiFi signal strength values.

- When partially labelled RSS data collected mobile devices and no location information of access points are available, the model performs similarly to LeMan [28], which is a semi-supervised algorithm for sensor-networkbased localization based on manifold learning. LeMan calibrates a tracking system purely from the client site.

- In general, when RSS data collected mobile devices and locations of access points are partially labelled, we can use all the available data for model building and get a better result than using part of the information only. We have studied how the labelled and unlabelled data help co-localization in [15], [18].

\section{Extension With Action Models}

As we describe in Section 1, localization systems can be classified into two categories: landmark-based and landmarkfree. Landmark-based systems rely on the measurement between a tracking target and multiple landmarks such as the received signal strength between a WiFi client and multiple access points. Landmark-free systems can localize themselves without the need of external references. An Inertial Navigation System can continuously update its position from measured velocity and time. Sensor readings may be inaccurate and noisy in either category of systems. WiFi signal has large noise in a complex indoor environment due to shadowing and multi-path effects. Inertial systems produce inaccurate dead reckoning over long periods, but accurately estimate relative motion over short intervals. In this section, we leverage the use of multiple sensors and extend the localization framework as learning Action Respecting Manifold (LARM for short).

\subsection{Problem Re-Statement}

Similar to the problem statement described in Section 3.1, assume that a user holds a mobile device and navigates in a two-dimensional indoor wireless environment $\mathcal{C} \subseteq \mathbb{R}^{2}$ with $n$ access points, which can periodically send out beacon signals. At some time $t_{i}$, the RSS values from all the $n$ access points are measured by the mobile device to form a row vector $\mathbf{s}_{i}=\left[\begin{array}{llll}s_{i 1} & s_{i 2} & \ldots & s_{i n}\end{array}\right] \in \mathbb{R}^{n}$. A sequence of $m$ signal strength vectors form an $m \times n$ matrix $S=\left[\begin{array}{llll}\mathbf{s}_{1}^{\prime} & \mathbf{s}_{2}^{\prime} & \ldots & \mathbf{s}_{m}^{\prime}\end{array}\right]^{\prime}$. Furthermore, the mobile device has additional sensors for measuring the activity of the mobile user. Such sensors can be compass or accelerometer, from which we can estimate the moving direction and speed. Let the speed at time $t_{i}$ be $o_{i}$ and the direction or azimuth be $\theta_{i}$. In this paper, azimuth is measured in angle in degree. It ranges in $\left[0^{\circ}, 360^{\circ}\right)$ and $0^{\circ}=$ North, $90^{\circ}=$ East, $180^{\circ}=$ South, $270^{\circ}=$ West. We denote $O=\left[\begin{array}{llll}o_{1} & o_{2} & \ldots & o_{m}\end{array}\right]^{\prime}$ and $\Theta=\left[\begin{array}{llll}\theta_{1} & \theta_{2} & \ldots & \theta_{m}\end{array}\right]^{\prime}$ column vectors of the sequences of speed and azimuth, respectively.

The locations of the mobile device at some time $t$ are labelled, while the rest are unlabelled. Furthermore, locations of some access points are known, while the rest are unknown. Our objective is to estimate the $m \times 2$ location matrix $P=\left[\mathbf{p}_{1}^{\prime}, \mathbf{p}_{2}^{\prime}, \ldots, \mathbf{p}_{m}^{\prime}\right]^{\prime}$ and $n \times 2$ location matrix $Q=\left[\mathbf{q}_{1}^{\prime}, \mathbf{q}_{2}^{\prime}, \ldots, \mathbf{q}_{n}^{\prime}\right]^{\prime}$, where $\mathbf{p}_{i}=\left[\begin{array}{ll}p_{i_{1}} & p_{i_{2}}\end{array}\right] \in \mathcal{C}$ and $\mathbf{q}_{j}=\left[\begin{array}{ll}q_{j_{1}} & q_{j_{2}}\end{array}\right] \in \mathcal{C}$ are the location of the mobile device at $t_{i}$ and the location of the $j^{t h}$ access point respectively.

Example 3 Again, Figure 1 shows an indoor WiFi environment with 5 access points deployed. A user holds a mobile device and walks from $A$ through $B, \ldots, E$ and finally stop at $F$ at time $t_{A}, t_{B}, \ldots, t_{F}$. Besides the signal strength vectors collected in Table 1, we can get azimuth vectors from the compass sensor e.g., $\Theta=\left[\begin{array}{llll}270^{\circ} & 180^{\circ} 90^{\circ} 0^{\circ} 180^{\circ} 90^{\circ} 90^{\circ}\end{array}\right]^{\prime}$, and estimate the walking speed from the accelerometer sensor. Assume the user walk at a constant speed $(1 \mathrm{~m} / \mathrm{s})$ and stops at $F$, the speed vector is estimated as $O=\left[\begin{array}{lllllll}1 & 1 & 1 & 1 & 1 & 1 & 0\end{array}\right]^{\prime}$. Our task is to estimate the trajectory matrix $P$ of the mobile device and the location matrix $Q$ of the access points.

\subsection{Signal Characteristics}

Besides all the domain characteristics described in subsection 3.2, there is one more important feature that explores the connection between actions and location changes:

- Consider two actions inferred from the motion sensors. If their actions are similar, the location change may also be similar. For example in Figure 1, a user walks from $A$ through $B, C, D, A, E$ to $F$ at time $t_{A}, t_{B}, t_{C}, t_{D}, t_{A^{\prime}}$, $t_{E}, t_{F}$. The actions of the mobile device are both move (east) at time $t_{C}$ and $t_{A^{\prime}}$, the change of their locations $\Delta C$ and $\Delta A^{\prime}$ should be similar. Note that the change of locations is a vector, having distance and direction of changes (Fig 5).

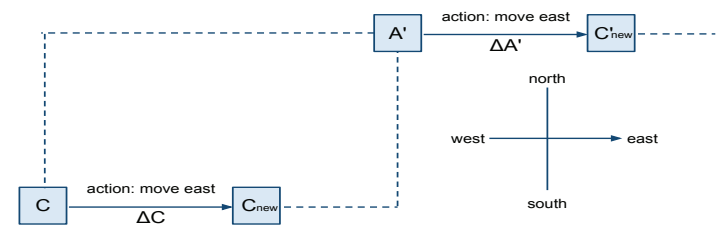

Fig. 5. Similar actions (move east) result in similar change of location $\left(\Delta C\right.$ and $\left.\Delta A^{\prime}\right)$.

\subsection{Dead Reckoning Localization}

Let the initial position, speed and azimuth of the mobile device be $\mathbf{p}_{1}, o_{1}$ and $\theta_{1}$. We set up a coordinate system using $\mathbf{p}_{1}$ as the origin, east as the positive $\mathrm{x}$-axis and north as the positive $\mathrm{y}$-axis. The location can be updated with $\mathbf{p}_{i+1}=\mathbf{p}_{i}+\Delta \mathbf{p}_{i}(i=$ 


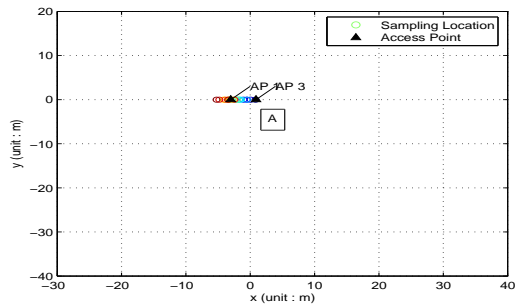

(a) Walk by A: detect $A P_{1}$ and $A P_{3}$

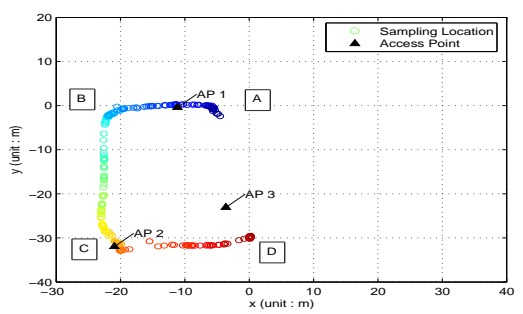

(d) Walk by $\mathrm{D}$ : revise $A P_{3}$

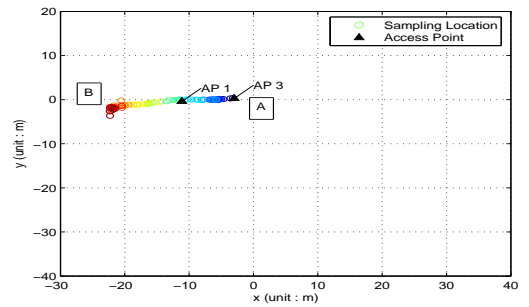

(b) Walk by $\mathrm{B}$ : revise $A P_{1}$

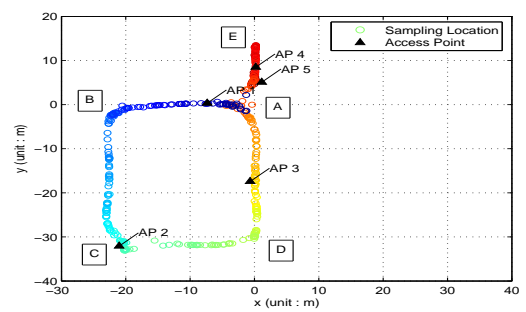

(e) Walk by E: detect $A P_{4}$ and $A P_{5}$

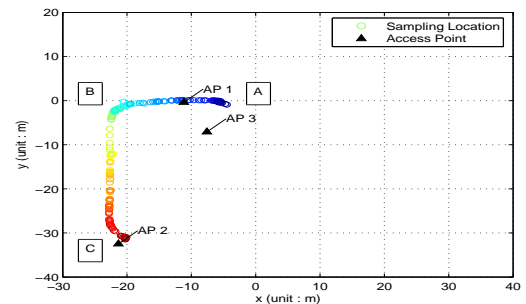

(c) Walk by $\mathrm{C}$ : detect $A P_{2}$ and revise $A P_{3}$

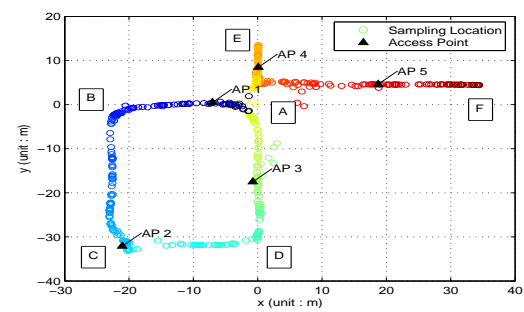

(f) Walk by $\mathrm{F}$ : revise $A P_{5}$

Fig. 4. Illustration of the online co-localization when a user walks from $A$ through $B, C, D, E$ to $F$.

$1, \ldots, m-1)$, where $\mathbf{p}_{i+1}$ and $\mathbf{p}_{i}$ are the locations at time $t_{i+1}$ and $t_{i} . \Delta \mathbf{p}_{i}$ is the displacement in the interval $\Delta t_{i}=t_{i+1}-t_{i}$. More specifically,

$$
\Delta \mathbf{p}_{i}=\left[\begin{array}{ll}
\Delta p_{i_{1}} & \Delta p_{i_{2}}
\end{array}\right]=\left[\begin{array}{c}
o_{i} * \Delta t_{i} * \sin \left(\theta_{i}\right) \\
o_{i} * \Delta t_{i} * \cos \left(\theta_{i}\right)
\end{array}\right]^{\prime},
$$

where $o_{i}$ and $\theta_{i}$ can be inferred from accelerometer and compass sensors, respectively.

Alternatively, we can reformulate it as an optimization problem. The objective is to minimize $\sum_{i=1}^{m-1}\left(\left(\mathbf{p}_{i+1}-\mathbf{p}_{i}\right)-\Delta \mathbf{p}_{i}\right)^{2}$. Again, we can rewrite it as a matrix form,

$$
P^{*}=\underset{P \in \mathbb{R}^{m \times 2}}{\arg \min }\left(G_{P} P-\Delta P\right)^{\prime} J_{\Delta P}\left(G_{P} P-\Delta P\right),
$$

where $G_{P}=\left(g_{i j}\right)_{m \times m}$. If $1 \leq i \leq m-1 \& i=j$, then $g_{i j}=1$, else if $1 \leq i \leq m-1 \& i=j-1, g_{i j}=-1$, otherwise $g_{i j}=0 . P$ is the coordinate matrix of the mobile device to be determined, and $J_{\Delta P}=\operatorname{diag}\left(\delta_{1}, \delta_{2}, \ldots, \delta_{m-1}, 0\right)$ is an indication matrix where $\delta_{i}=1$ if the action information of the mobile user at time $t_{i}$ is available and otherwise $\delta_{i}=0$, and $\Delta P=\left[\begin{array}{lllll}\Delta \mathbf{p}_{1}^{\prime} & \Delta \mathbf{p}_{2}^{\prime} & \ldots & \Delta \mathbf{p}_{m-1}^{\prime} \mathbf{0}^{\prime}\end{array}\right]^{\prime}$ is an $m \times 2$ matrix supplying the calibration data where $\Delta \mathbf{p}_{i}$ is the change of location from time $t_{i}$ to $t_{i+1}$ if $\delta_{i}=1$ and otherwise the value of $\Delta \mathbf{p}_{i}$ can be any. By setting the derivative of the right hand side in the optimization problem (14) to zero, we can get a close form solution $P=\left(G_{P}^{\prime} J_{\Delta P} G_{P}\right)^{-1} G_{P}^{\prime} J_{\Delta P} \Delta P$.

Note that matrix $G_{P}$ is singular, and thus matrix inverse is not applicable. One may consider using pseudo-inverses. Alternatively, we can pose a regularization term $P^{\prime} P$ to the objective function as many machine learning methods do. Meanwhile, we borrow the idea from Action Respecting Embedding [22] and add another term $P^{\prime} G_{P}^{\prime} L_{\Delta P} G_{P} P$ to measure the smoothness of actions. Then we get a new optimization problem as:

$$
\begin{gathered}
P^{*}=\underset{P \in \mathbb{R}^{m \times 2}}{\arg \min } \quad \alpha\left(G_{P} P-\Delta P\right)^{\prime} J_{\Delta P}\left(G_{P} P-\Delta P\right) \\
+\beta P^{\prime} G_{P}^{\prime} L_{\Delta P} G_{P} P+\epsilon P^{\prime} P,
\end{gathered}
$$

where $\alpha, \beta$ and $\epsilon$ are parameters to balance the loss function term, the smoothness of actions and the "complexity" of $P$, respectively. $L_{\Delta P}$ is the Graph Laplacian for describing the similarity of action pairs. Again, the similarity is described with Gaussian Kernel: $w_{i j}=\exp \left(-\left\|\boldsymbol{\Delta}_{i}-\boldsymbol{\Delta} \mathbf{p}_{j}\right\|^{2} / 2 \sigma_{\Delta P}^{2}\right)$. By setting the derivative of the right hand side in (15) to zero, we can get a close form solution

Example 4 Figure 6(a) shows that a user holds a mobile device and walks in an area of $70 \mathrm{~m} \times 80 \mathrm{~m}$ from point $1,2, \ldots$, to 5. The device can measure the WiFi signal strength from the surrounding access points periodically. Meanwhile, the mobile device has digital compass and accelerometer sensors mounted so that we can estimate a sequence of azimuth $\theta_{i}$ and speed $o_{i}$, which will be converted to $\Delta \mathbf{p}_{i}$ using Equation (13). The localization result is obtained by solving (15). Figure 6(b) illustrates the localization trajectory. Compared to the ground truth trajectory shown in Figure 6(a), the estimated locations are accurate at an initial stage, say from point 1 to 2 . It gradually becomes inaccurate because the error is accumulated as time elapses. Note that, we use an uncalibrated compass for collecting data. Therefore, the azimuth reading may not be accurate. However, a calibrated compass may be disturbed and become inaccurate if it is close to some local magnetic fields such as elevators.

\subsection{Extension: The LARM Algorithm}

By combing the dead reckoning objective (15) and the manifold-based objective (2) together, we optimize:

$$
\begin{aligned}
P^{*}=\underset{P \in \mathbb{R}^{m \times 2}}{\arg \min } & \left(P-Y_{P}\right)^{\prime} J_{P}\left(P-Y_{P}\right)+\beta P^{\prime} G_{P}^{\prime} L_{\Delta P} G_{P} P+\epsilon P^{\prime} P \\
& +\gamma_{P} P^{\prime} L_{P} P+\alpha\left(G_{P} P-\Delta P\right)^{\prime} J_{\Delta P}\left(G_{P} P-\Delta P\right) .
\end{aligned}
$$

The first term is the fitting error to labelled data. The second term describes the smoothness on the action manifold. The third term poses a penalty on the complexity of the solution. The fourth term is the smoothness on the signal manifold. The fifth term is the agreement of location changes. 


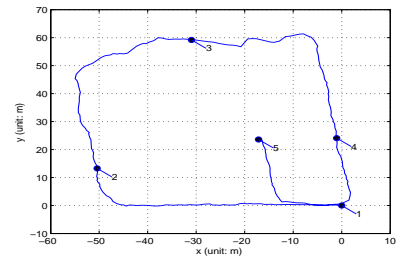

(a) Ground truth trajectory

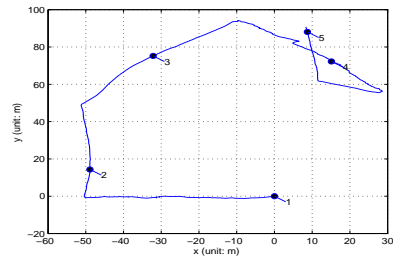

(b) Dead reckoning trajectory

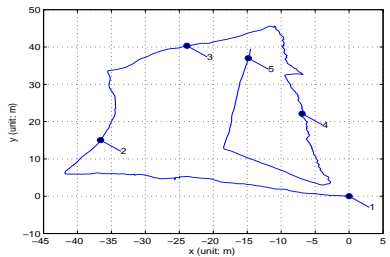

(c) Unsupervised ARM

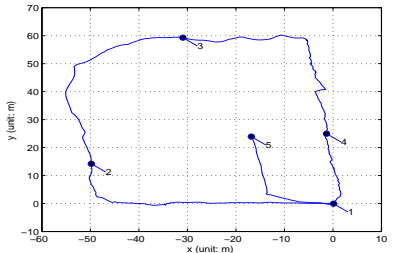

(d) Semi-supervised ARM

Fig. 6. Localization comparison with motion sensors.

Example 5 By combining all sensor readings together, we can recover a better location map for either unsupervised or semi-supervised cases. Figure 6(c) shows the localization result without any labelled location. If we compare Figure 6(c) to Figure 6(b), we can see that unsupervised LARM can greatly correct the drifting error of Dead Reckoning by using additional unlabelled WiFi signal data. Additionally, if we have 5 percent of random labelled locations, the recovered trajectory as shown in Figure 6(d) will be pretty close to the ground truth.

Note that a motion or action model is unavailable for access points. Thus, we can combine (15) with (7) or (9) and form the objective of action-guided co-localization. The essential idea is to incorporate possible constraints about the similarity among signals and locations of mobile devices and access points, location changes and actions, etc. The new optimization problem of action guided co-localization can be written as follows,

$$
\begin{aligned}
R^{*}=\underset{R \in \mathbb{R}^{(m+n) \times 2}}{\arg \min } & (R-Y)^{\prime} J(R-Y)+\gamma R^{\prime} L R+\beta R^{\prime} G^{\prime} L_{\Delta R} G R \\
+ & \alpha(G R-\Delta R)^{\prime} J_{\Delta R}(G R-\Delta R)+\epsilon R^{\prime} R,
\end{aligned}
$$

where $R=\left[\begin{array}{ll}P^{\prime} & Q^{\prime}\end{array}\right]^{\prime}$ is the coordinate matrix of the mobile device and the access points; $Y=\left[\begin{array}{ll}Y_{P}^{\prime} & Y_{Q}^{\prime}\end{array}\right]^{\prime}$ gives the label information; $J=\left[\begin{array}{cc}J_{P} & 0 \\ 0 & J_{Q}\end{array}\right]$ is the indication matrix. $L=\gamma_{A} L_{A}+$ $\gamma_{B} L_{B}+\gamma_{C} L_{C}$, which is the same as defined in Section 3.5. $G=\left[\begin{array}{ll}G_{P}^{\prime} & \mathbf{0}^{\prime}\end{array}\right]^{\prime}, L_{\Delta R}=\left[\begin{array}{ll}L_{\Delta P}^{\prime} & \mathbf{0}^{\prime}\end{array}\right]^{\prime}, \Delta R=\left[\begin{array}{ll}\Delta P^{\prime} & \mathbf{0}^{\prime}\end{array}\right]^{\prime}$ and $J_{\Delta R}=$ $\left[\begin{array}{ll}J_{\Delta P}^{\prime} & \mathbf{0}^{\prime}\end{array}\right]^{\prime}$. Similarly, a close form solution of the optimization problem can be obtained by

$$
\begin{aligned}
R= & \left(J+\gamma L+\alpha G^{\prime} J_{\Delta R} G+\beta G^{\prime} L_{\Delta R} G+\epsilon I\right)^{-1} \\
& \times\left(J Y+\alpha G^{\prime} J_{\Delta R} \Delta R\right) .
\end{aligned}
$$

Note that the above solution still works even if all locations are unlabelled and actions are partially labelled.

\subsection{Action Recognition}

In previous sections, we assume the walking speed and direction can be estimated from accelerometer and compass sensors. While it is straight-forward to obtain direction readings $\Theta=\left[\theta_{1}, \theta_{2}, \ldots, \theta_{m}\right]^{\prime}$ from compass sensors, we have not yet described any detail on how to estimate the speed $O=\left[o_{1}, o_{2}, \ldots, o_{m}\right]^{\prime}$. Whether a user is running, walking or standing still can be inferred from accelerometer sensors. When we recognize that the user is running or walking, the speed can be estimated via step counting, assuming the step size is known and fixed.
To recognize the user actions, we transform the sequential accelerometer data into a fixed dimension of features. More specifically, we apply a sliding window on the signals and extract the mean value, standard deviation, Cepstrum (a feature widely used in speech recognition) on each dimension of readings and Pearson correlation between pairs of dimensions. More features and models can be found at many previous works [34]. We collect a set of data and train a Support Vector Machine with Linear Kernel for action recognition. Table 2 shows the experimental results for six different actions from a different set of accelerometer data. The overall accuracy is $88.55 \%$. Note that the action "turn left" or "turn right" may be ambiguous while the action "static" is easy to be recognized. In practice, we do not need to recognize the actions on "turning" since compass sensor has richer information.

We further count the steps once we recognize that the user is walking or climbing upstairs/downstairs. To properly segment steps, we implement some cycle detection algorithm. We apply Fast Fourier Transformation (FFT) to detect whether there is a cycle in frequency domain. FFT is an efficient method for transforming signals from time domain to frequency domain[35]. By applying FFT, we can detect a strong cycle signal in the frequency domain based on periodic patterns. Assume that the user has a fixed step size, we can estimate the walking speed reasonably well.

TABLE 2

Action recognition using accelerometer sensor only

\begin{tabular}{|l|r|r|r|r|r|r|}
\hline $\begin{array}{l}\text { estimation } \\
\text { / truth }\end{array}$ & static & upstairs & downstairs & walk & left turn & right turn \\
\hline static & 994 & 0 & 0 & 1 & 7 & 6 \\
\hline upstairs & 0 & 959 & 102 & 115 & 42 & 5 \\
\hline downstairs & 0 & 36 & 967 & 56 & 34 & 9 \\
\hline walk & 0 & 1 & 0 & 4457 & 112 & 131 \\
\hline left turn & 0 & 50 & 1 & 117 & 948 & 15 \\
\hline right turn & 0 & 0 & 5 & 248 & 24 & 314 \\
\hline
\end{tabular}

\section{Experimental Setup}

In this section, we first evaluate the performance of the colocalization algorithms on three sets of different devices and test-beds. They are wireless local area networks (WLAN), wireless sensor networks (WSN) and radio frequency identification networks (RFID). In the past, researchers have tried to formalize various metrics for evaluating activity recognition and location based services [36]. A summary of our three experimental setups is shown in Table 3. 
TABLE 3

The experimental setups of WLAN, WSN and RFID

\begin{tabular}{|c|c|c|c|c|c|}
\hline & AP & MD & test-bed & scale & motion pattern \\
\hline WLAN & 25 APs & 1 notebook & hallway & $60 \times 50 \mathrm{~m}^{2}$ & mobile (human) \\
\hline WSN & 8 nodes & 1 mobile node & room & $5 \times 4 m^{2}$ & mobile (robot) \\
\hline RFID & 4 readers & 30 RFID tags & room & $5 \times 4 m^{2}$ & static \\
\hline
\end{tabular}

A person carrying an $\mathrm{IBM}^{\circledR} \mathrm{T} 42$ notebook, which is equipped an Intel ${ }^{\complement}$ Pro/2200GB internal wireless card, walks in an indoor environment of about $60 \mathrm{~m} \times 50 \mathrm{~m}$ in size. An IEEE $802.11 \mathrm{~b}$ wireless network in the $2.4 \mathrm{GHz}$ frequency bandwidth has been set up in the indoor environment. We can detect more than 20 access points. The person walks in the hallways and a total of 2000 examples (vectors of RSS values) are collected with sample rate $2 \mathrm{~Hz}$. The ground-truth location labels are obtained by referring to landmark points such as doors, corners and dead-ends. The localization area is composed by one-dimensional hallways.

The sensor-based tracking experiment is performed in the Pervasive Computing Laboratory at the Hong Kong University of Science and Technology. The room is set up an experimental test-bed of 5.0 meter by 4.0 meter.

We use CrossBow MICA2 and MICA2Dot to construct a wireless sensor network. We program these sensor nodes to broadcast and detect beacon frames periodically so that they can measure the RSS from each other. By combining the RSS from different nodes we can estimate locations of these nodes. We configure all the nodes such that each of them can measure the RSS from the remaining eight nodes in every $0.5 \mathrm{~s}$. We try different kinds of robots that could run freely around the floor such as Sony AIBO dogs, LEGO Mindstorms and off-theshelf toy cars. A Camera Array is used to record experiments for supporting location information (ground truth) of mobile robots. Each camera monitors at least one-fourth part of the test-bed. The central area is covered by all four cameras. We use some landmarks to do camera calibrationsuch as static sensor nodes which locations are known.

For the RFID experiment, we used four Mantis readers (AP) and 30 tags (MD) from RF Code ${ }^{\circledR}$. They are all deployed as stationary nodes. All the tags are deployed at $6 \times 5$ grid points on the $5.0 \mathrm{~m} \times 4.0 \mathrm{~m}$ floor. A total of 2,000 examples with ground truth locations were collected.

\section{EXPERIMENTAL RESULtS}

\subsection{Accuracy Test of Two-Phase Co-Localization}

For comparison, we run the following baseline algorithms (1) LANDMARC, a nearest-neighbor weighting based method designed for RFID localization [37]; (2) Support Vector Regression (SVR), a simplified variant of a kernel-based method used for WSN localization [11]; (3) RADAR, a KNN method for WLAN localization [9]. In each experiment, we randomly picked 500 examples for training and the rest for testing. The training data was further split into labelled and unlabelled parts. The results shown in Figure 7 are averaged over 10 repetitions for reducing statistical variability. All results are measured in relative error distances, which are error distances in percentage while referring to the maximal error distance in each figure for easy comparison.

LANDMARC, RADAR and SVR were trained with the labelled part of training data. In contrast, the proposed twophase co-localization method uses both labelled and unlabelled data. We test on two configurations for the two-phase co-localization method: (1) "Co-Localization no AP" uses partially labelled data from mobile devices for training, in which we try to recover the locations of the access points; and (2) "Co-Localization with AP" repeats the same experiments with the locations of all access points known. Note that all error distances are presented in percentage since they are normalized when referring to the maximal error in each figure.

\subsubsection{Model Parameter Setting}

Our experiments mainly target at showing how labelled and unlabelled data can help increase accuracy and reduce calibration effort in relative error distance. We do not specifically fine tune the parameters. Instead, parameters are determined in a validation set at a coarse level. We set $s^{\max }=-30 \mathrm{dBm}$ and $\sigma=8$ in the Gaussian function in Equation (1) for signals in all networks. $s^{\max }=-30$ is a value that roughly ranks top $1 \%$ of all signal strength values. We avoid using top $1^{\text {st }}$ value to avoid potential outlier. We also try other values in the experiment such as $s^{\max }=-40$ and $\sigma=16$. There is no significant difference in the experiment results. We use $k$ nearest neighbors for building the neighborhood graph in constructing all graph Laplacians. We set $k=10$ for $L_{P}$ in Equation (3) and $k=5$ for $L_{Q}$ in Equation (4) after trying popular values such as 5,10 or 15 in manifold learning. $\gamma$ is a global regularization term for second level terms $\gamma_{A}$, $\gamma_{B}, \gamma_{C}$ in Equation (8). In the following experiments, we set $\gamma_{A}=0.01, \gamma_{B}=1.0, \gamma_{C}=0.001$ and $\gamma=0.0001$, which are tuned in a validation set. The details of a strategy of parameter $(\gamma$ 's) tuning and a sensitivity study on the parameters will be described in Section 6.1.3.

\subsubsection{Comparison Results}

Figures 7(a), 7(c) and 7(e) show the location estimation errors of different mobile devices by varying the number of labelled examples in a training set whose size is fixed to be 500 . We can observe from the figures that firstly, if we compare the results vertically in each figure, we can see how the unlabelled data help improve the result in the proposed methods. For example in Figure 7(e), most compared methods have a relative error distance of around $80 \%$ when using 50 labelled examples. In contrast, the proposed methods have an error of around $40 \%$ by employing additional 450 unlabelled examples. Secondly, if we compare the results horizontally in each figure, we can find how our methods reduce calibration effort. For example in Figure 7(a), most compared methods have a relative error distance of around $60 \%$ when all 500 examples are labelled. The "Co-Localization with AP" has similar performance when using only 50 labelled and 450 unlabelled examples. Hence, we save the calibration effort dramatically.

We can find that the mobility of the mobile device and the environment complexity are two main factors that affected the performance of the two-phase co-localization algorithm. 


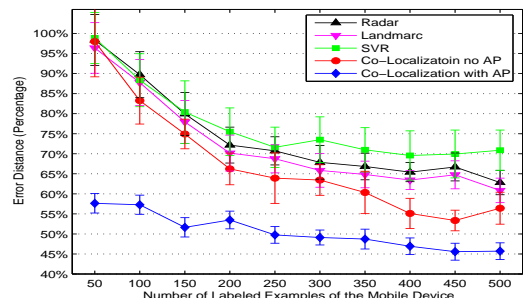

(a) RFID MD (tags)

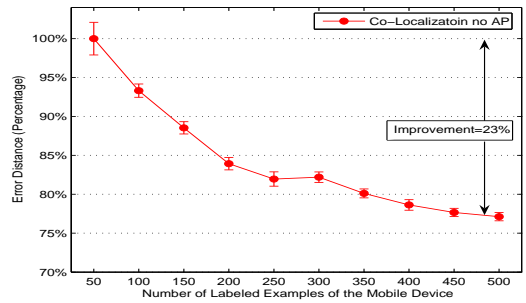

(d) WSN AP (static sensor nodes)

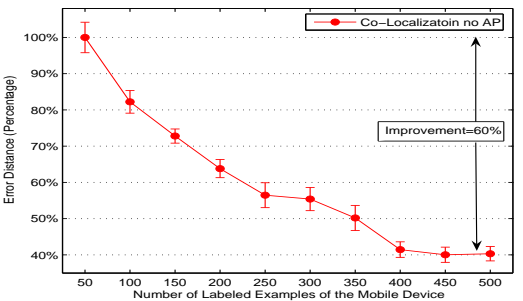

(b) RFID AP (readers)

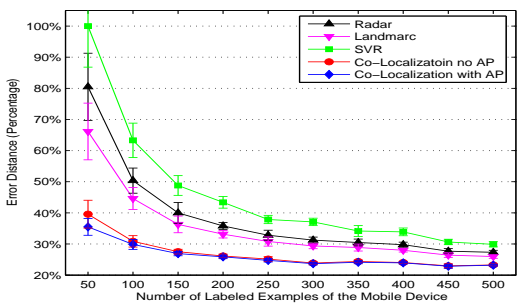

(e) WLAN MD (notebook)

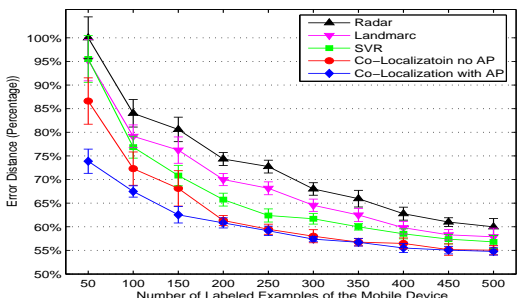

(c) WSN MD (mobile sensor node)

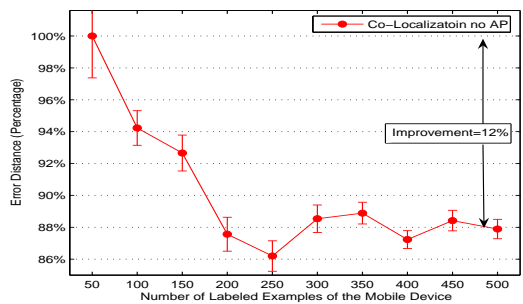

(f) WLAN AP (access points)

Fig. 7. Experimental results over 10 repetitions (Mean and Std.): MD for mobile device; AP for access point.

In a static and plane-shaped test-bed (Figure 7(a)), the radio signals are less noisy and the "Co-Localization no AP" configuration demonstrated similar performance as RADAR, LANDMARC and SVR when the number of labelled examples is small. In a mobile and complex environment, as shown in (Figure 7(e)), the radio signal is more noisy and the "CoLocalization no AP" performed much better and more robust than the compared methods. We have also tried some other combinations of experiments that led to a similar conclusion, such as using RFIDs in a mobile scenario.

While comparing the results of "Co-Localization no AP" and "Co-Localization with AP" in Figures 7(a), 7(c) and 7(e), we can find that knowing the locations of access points is more helpful for localizing the mobile devices in a static and planar scenario (Figure 7(a)) than in a mobile and complex environment (see Figure 7(e)). Similarly, we can see from Figures 7(b), 7(d) and 7(f) that knowing the locations of mobile devices are more helpful for localizing access points in a static and plane-shaped scenario rather than a mobile and complex environment.

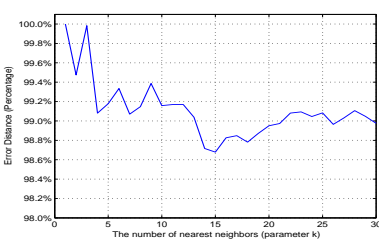

(a) Change values of $k$

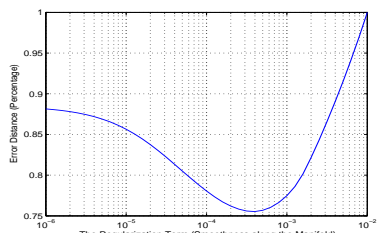

(b) Change the regularization term
Fig. 8. Parameter Tuning on a Validation Set.

\subsubsection{Parameter Sensitivity Test}

In this section, we study the parameter sensitivity on the performance of the two-phase co-localization algorithm. Figure 8 shows the sensitivity of relative error distances while varying parameters such as the $k$ for retrieving top nearest neighbors in Figure 8(a) and the regularization parameters $\gamma$ for penalizing the smoothness along the data manifold in Figure 8(b) on a validation set. As can be seen from Figure $8(a)$, the error ranges in $[98.5 \%, 99.4 \%]$ (less than $1 \%$ change) when $k$ varies from 5 to 20 . This observation is consistent with most manifold-based learning methods when $k$ is generally picked up among popular values such as 5 , 10 or 15 . Besides the parameter $k$, there are several $\gamma$ 's for controlling the smoothness on data manifolds, $\gamma_{A}, \gamma_{B}, \gamma_{C}$ and $\gamma$. As we described in Section 6.1.1, $\gamma$ is a global tuning term for $\gamma_{A}, \gamma_{B}$ and $\gamma_{C}$ in Equation (8). For tuning these parameters in a validation set, we first fix $\gamma_{B}$ to 1 and tune the others. The tuning strategy may not be optimal but it works well in our experiments. Figure 8(b) shows how the error changes when

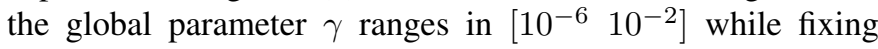
$\gamma_{B}=1, \gamma_{A}=0.01$ and $\gamma_{C}=0.001$. The best value can be picked up in about $\left[10^{-4} 10^{-3}\right]$. Small changes in parameter setting such as $k$ and $\gamma$ would not change the trend of the curves shown in Figure 7.

\subsection{Speed Test of Online Co-Localization}

Figure 9 shows the average running time for adding a new training example. The test is done in Matlab on a computer with a $2.0 \mathrm{GHz}$ CPU. Experimental results show that we can greatly reduce the time for the model adaption in an online manner. For example, when the training dataset size is incrementally enlarged to about 500 , the two-phase method needs $1.2 s$ to re-estimate everything while the online method spends no more than $0.1 \mathrm{~s}$. The online method is more than ten times faster. The localization accuracy of the online model is similar to the two-phase counterpart. The difference is that the neighborhood graph and weight matrix are revised incrementally rather than rebuilt.

\subsection{Encode a Motion Model}

In this experiment, we aim to verify that, by employing a Kalman Filter, the error distances of all previous compared methods can be further reduced by $5 \%$ to $10 \%$ in a mobile 


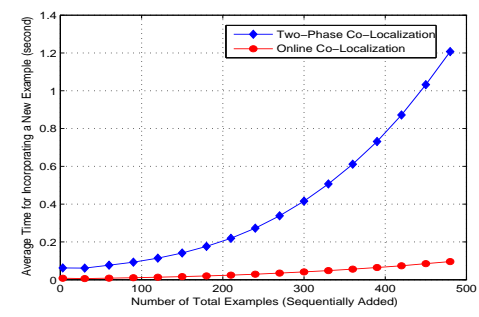

Fig. 9. Average running time comparison.

scenario. Here, we perform an additional three-dimensional tracking experiment, showing the usefulness of encoding motion models. The whole test-bed fills up a cubic space of $6.0 m \times 6.0 m \times 2.0 m$. In the test-bed, we have ten static nodes that send out beacon signals. Five of them are deployed on the floor and the rest on the ceiling. There is one more node that moves freely around the environment for tracking experiments. The ground truth location of the mobile node is exported from four cameras deployed in the laboratory. In Figure 10(a), the location of the mobile node is obtained by computing the intersection point $E$ of lines $C D$ and $A B$ estimated from two different cameras. We collect 1,000 examples, which are split into two parts: 500 examples for training and the rest for testing. Again we vary the number of labelled examples and repeat the experiments 10 times. The experimental result is shown in Figure 10(b). As can be seen, the error distance of co-localization with a motion model is about $10 \%$ smaller than that without a motion model when sufficient labelled data are available.

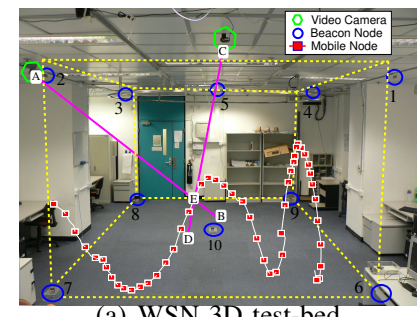

(a) WSN 3D test-bed

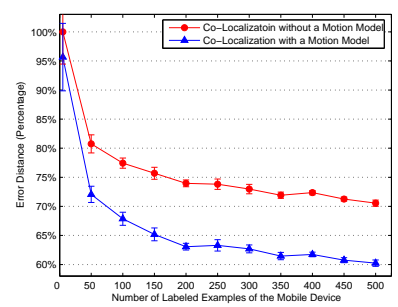

(b) Result of motion model test
Fig. 10. Experiments with a motion model.

\subsection{Encode an Action Model}

In Section 4.4, we have already demonstrated how LARM works in previous examples shown in Figure 6. In this section, we conduct experiments to study whether the LARM algorithm can further boost the performance of location tracking by fusing different types of sensors. For this purpose, we use an Android G1 phone for collecting data because it has a lot of built-in sensors such as accelerometer, magnetic sensors, WiFi, etc.

With a built-in 3-axis accelerometer sensor, system-detected orientation and direction can be directly read from a built-in compass sensor, and speed values can be indirectly estimated from the accelerometer sensors. Specifically, we applied the estimation method introduced in [34] to infer the speed from accelerometer readings. Since speed and direction values are taken as additional input, in our experiments, we did not explicitly vart the accuracy of speed/direction values. Instead, we focused on studying how the LARM algorithm can improve the localization accuracy by embedding an action model even when the speed and direction values contain noise.

The values (unit: $m / s^{2}$ ) are in the format of (X, Y, Z). The $\mathrm{X}$ axis refers to the screen's horizontal axis (the small edge in portrait mode, the long edge in landscape mode) and points to the right. The Y axis refers to the screen's vertical axis and points towards the top of the screen. The $\mathrm{Z}$ axis points toward the sky when the device is lying on its back on a table. Figure 11(a) shows the directions of $\mathrm{X}, \mathrm{Y}$ and $\mathrm{Z}$ when the phone works in portrait mode. Accelerometer sensor can be used as a pedometer for speed estimation. Orientation is estimated from a built-in compass sensor. It is represented by a triple (Azimuth, Pitch, Roll). All values are angles in degree. Azimuth is the rotation around the $\mathrm{Z}$ axis $\left(0^{\circ} \leq\right.$ azimuth $\left.<360^{\circ}\right)$, for which $0^{\circ}=$ North, $90^{\circ}=$ East, $180^{\circ}=$ South, $270^{\circ}=$ West as shown in Figure 11(a). Pitch is the rotation around $\mathrm{X}$ axis $(-180 \leq$ pitch $\leq 180)$, with positive values when the $\mathrm{Z}$-axis moves toward the Y-axis. Roll is the rotation around $\mathrm{Y}$ axis $(-90 \leq$ roll $\leq 90)$, with positive values when the $\mathrm{Z}$-axis moves toward the $\mathrm{X}$-axis.

Alignment among sensors are necessary during data collection because they have different sample rates. The rate of sampling WiFi signal strength is $2 \mathrm{~Hz}$. Accelerometer and orientation sensors have a sample rate $45 \mathrm{~Hz}$ and thus will be downsampled after action recognition naturally. In the area shown in Figure 6(a), we walk around and collect 1000 examples at sample rate $2 \mathrm{~Hz}$.

\subsubsection{Overall Results}

In each experiment, we randomly picked a small portion of data for labelling while the rest for testing. The results (mean error and standard deviation) shown in Figure 11(b) are averaged over 10 repetitions. The horizontal axis is the percentage of data that are labelled, which ranges from $0 \%$ to $10 \%$. The vertical axis is the average error distance in meters. As can be seen, Dead Reckoning Localization without using any labelled data has a large error due to drifting factor. When we combine WiFi tracking through co-localization and Dead Reckoning Localization together using unsupervised LARM, the error is reduced at least by half. If some small percent of labelled data are available, the location estimation error of the semi-supervised LARM algorithm can be reduced significantly. The fusion of WiFi and motion sensors with LARM also has better performance than using partially labelled WiFi data alone (denoted "Co-Localization without an Action Model").
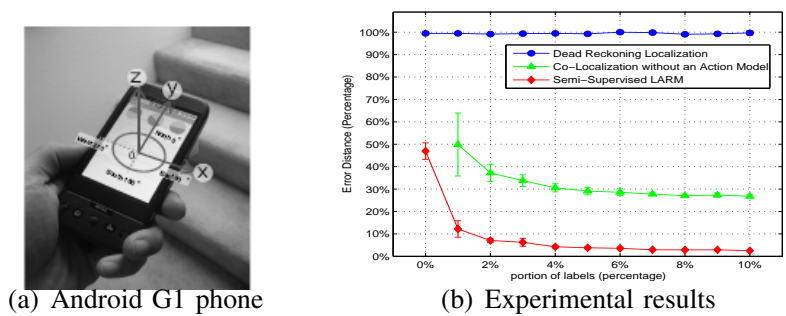

(b) Experimental results

Fig. 11. Experiments with an action model. 


\subsubsection{Parameter Sensitivity Test}

Compared to the two-phase co-localization algorithm, the action-model-embedded algorithm LARM has three more parameters $\alpha, \beta$ and $\epsilon$. In experiments shown in the previous section, we use the same parameter settings for $\gamma_{A}, \gamma_{B}, \gamma_{C}, \gamma$ and $k$ as described in Section 6.1.3, and set $\alpha=0.5, \beta=0.001$ and $\epsilon=10^{-6}$ in Equation (16), which are all tuned in a validation set. In this section, we fix the values of $\gamma_{A}, \gamma_{B}$, $\gamma_{C}, \gamma$ and $k$ to test the sensitivity at different values of $\alpha, \beta$ and $\epsilon$ on the overall performance of LARM. Figure 12 shows how the error distance changes while looping through $\alpha, \beta$ and $\epsilon$. As can be seen in Figure 12(a), LARM performs well when $\alpha$ ranges in $\left[10^{-1} 10^{1}\right]$. Similarly, Figure 12 (b) suggests that the best value for $\beta$ falls in the range $\left[10^{-4} 10^{-2}\right]$. Figure 12 (c) shows that the performance of LARM is good and vary little when $\epsilon$ is set to a value smaller than $10^{-4}$.

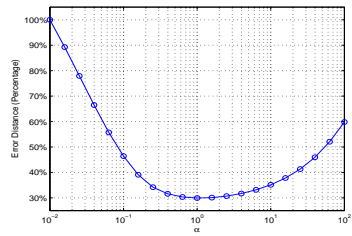

(a) Change values of $\alpha$

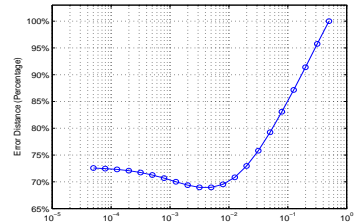

(b) Change values of $\beta$

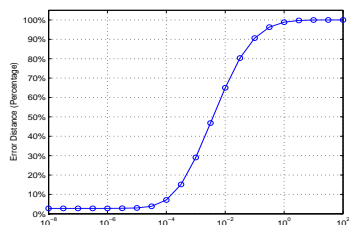

(c) Change values of $\epsilon$

Fig. 12. Parameter sensitivity study with an action model.

\section{Conclusion And Future Works}

In this paper, we proposed a novel semi-supervised graph Laplacian approach to solve the problem of simultaneously recovering the locations of both mobile devices and access points. In our co-localization framework, we estimated the relative locations of mobile devices and access points by exploiting a SVD based method, and estimated the absolute locations using a small collection of labelled data through graph Laplacian methods. Our extensive experiments in three different test-beds showed that we can achieve high performance with much less calibration effort as compared to several previous approaches. Meanwhile, our model can deal with data stream and adjust itself online relatively faster while compared to its two-phase counterpart. Finally, we extend our framework for multiple sensor fusion via an Action Respecting Manifold. Several demonstrations and experimental results show that the performance of combing multiple sensors for localization is much better than using them individually.

The significance of the work is that we can leverage the knowledge of the access point locations, the mobile device trajectories and motion sensors to obtain more accurate localization. We will continue to evaluate the performance in a large-scale and dynamic environment, e.g., in a city level and at different time periods. We may also vary more parameters such as the number of access points and their deployment density and study the robustness of our proposed algorithm.

AcknowledgementWe thank Seth Teller, Rong Pan, Vincent Wenchen Zheng for their helpful discussions and ideas during the long run of this work in the past years. We also thank the support of Hong Kong RGC/NSFC N_HKUST624/09 and Hong Kong RGC grant 621010, and Microsoft Research Asia Grant MRA10EG01.

\section{REFERENCES}

[1] L. Liao, D. J. Patterson, D. Fox, and H. A. Kautz, "Learning and inferring transportation routines," Artificial Intelligence, vol. 171, no. 5-6, pp. 311-331, 2007.

[2] Y. Zheng and X. Xie, "Learning travel recommendations from usergenerated gps traces," ACM Trans. Intell. Syst. Technol., vol. 2, pp. 2:12:29, January 2011.

[3] K. Farrahi and D. Gatica-Perez, "Discovering routines from large-scale human locations using probabilistic topic models," ACM Trans. Intell. Syst. Technol., vol. 2, pp. 3:1-3:27, January 2011.

[4] M. A. Batalin, G. S. Sukhatme, and M. Hattig, "Mobile robot navigation using a sensor network," in IEEE International Conference on Robotics and Automation. IEEE, April 2004, pp. 636-642.

[5] S. Thrun, W. Burgard, and D. Fox, Probabilistic Robotics. Cambridge, MA: MIT Press, September 2005.

[6] A. Kotanen, M. Hannikainen, H. Leppakoski, and T. D. Hamalainen, "Positioning with IEEE 802.11b wireless LAN," in Proceedings of the 14th IEEE Proceedings on Indoor and Mobile Radio Communications. IEEE, September 2003, pp. 2218-2222.

[7] Q. Yang, S. J. Pan, and V. W. Zheng, "Estimating location using Wi-Fi," IEEE Intelligent Systems, vol. 23, no. 1, pp. 8-13, 2008.

[8] D. Maligan, E. Elnahrawy, R. Martin, W. Ju, P. Krishnan, and A. S. Krishnakumar, "Bayesian indoor positioning systems," in Proceedings of the 24th IEEE International Conference on Computer Communications, Joint Conference of the IEEE Computer and Communications Societies. IEEE, March 2005, pp. 1217-1227.

[9] P. Bahl and V. Padmanabhan, "RADAR: An in-building RF-based user location and tracking system," in Proceedings of the 19th IEEE International Conference on Computer Communications, Joint Conference of the IEEE Computer and Communications Societies, vol. 2, March 2000 pp. 775-784.

[10] B. Ferris, D. Hahnel, and D. Fox, "Gaussian processes for signal strength-based location estimation," in Proceedings of Robotics: Science and Systems. MIT Press, August 2006.

[11] X. Nguyen, M. I. Jordan, and B. Sinopoli, "A kernel-based learning approach to ad hoc sensor network localization," ACM Transactions on Sensor Networks, vol. 1, no. 1, pp. 134-152, 2005.

[12] S. C. Deerwester, S. T. Dumais, T. K. Landauer, G. W. Furnas, and R. A. Harshman, "Indexing by latent semantic analysis," Journal of the American Society of Information Science, vol. 41, no. 6, pp. 391-407, 1990.

[13] M. Belkin and P. Niyogi, "Laplacian eigenmaps for dimensionality reduction and data representation," Neural Computation, vol. 15, no. 6, pp. 1373-1396, 2003.

[14] J. Ham, D. Lee, and L. Saul, "Semisupervised alignment of manifolds," in Proceedings of the 10th International Workshop on Artificial Intelligence and Statistics. Society for Artificial Intelligence and Statistics, January 2005, pp. 120-127.

[15] J. J. Pan and Q. Yang, "Co-localization from labeled and unlabeled data using graph laplacian," in Proceedings of the 20th International Joint Conference on Artificial Intelligence. Morgan Kaufmann Publishers, 2007, pp. 2166-2171.

[16] M. H. C. Law and A. K. Jain, "Incremental nonlinear dimensionality reduction by manifold learning," IEEE Transaction on Pattern Analysis and Machine Intelligence, vol. 28, no. 3, pp. 377-391, 2006.

[17] O. Kouropteva, O. Okun, and M. Pietikainen, "Incremental locally linear embedding algorithm," Pattern Recognition, vol. 38, no. 10, pp. 1764 1767, October 2005.

[18] J. J. Pan, Q. Yang, and S. J. Pan, "Online co-localization in indoor wireless networks by dimension reduction," in Proceedings of the 22nd National Conference on Artificial Intelligence. AAAI Press, 2007, pp. 1102-1107 
[19] B. Ferris, D. Fox, and N. Lawrence, "WiFi-SLAM using gaussian process latent variable models," in Proceedings of the 20th International Joint Conference on Artificial Intelligence. Hyderabad, India: Morgan Kaufmann Publishers Inc., 2007, pp. 2480-2485.

[20] T. Yairi, "Map building without localization by dimensionality reduction techniques," in Proceedings of the 24th international conference on Machine learning. ACM Press, 2007, pp. 1071-1078.

[21] E. Foxlin, "Pedestrian tracking with shoe-mounted inertial sensors," IEEE Computer Graphics and Applications, vol. 25, no. 6, pp. 38-46, 2005.

[22] M. Bowling, A. Ghodsi, and D. Wilkinson, "Action respecting embedding," in Proceedings of the 22nd International Conference on Machine Learning. ACM Press, 2005, pp. 65-72.

[23] J. Letchner, D. Fox, and A. LaMarca, "Large-scale localization from wireless signal strength," in Proceedings of the 20th National Conference on Artificial Intelligence. AAAI Press / MIT Press, July 2005, pp. 1520.

[24] M. Youssef, A. Agrawala, and U. Shankar, "WLAN location determination via clustering and probability distributions," in Proceedings of the First IEEE International Conference on Pervasive Computing and Communications. IEEE, March 2003, pp. 143-150.

[25] S. J. Pan and Q. Yang, "A survey on transfer learning," IEEE Transactions on Knowledge and Data Engineering, vol. 22, no. 10, pp. 13451359, October 2010.

[26] J. Shawe-Taylor and N. Cristianini, Kernel Methods for Pattern Analysis. New York, NY, USA: Cambridge University Press, 2004.

[27] M. Belkin, P. Niyogi, and V. Sindhwani, "Manifold regularization: A geometric framework for learning from labeled and unlabeled examples," Journal of Machine Learning Research, vol. 7, pp. 2399-2434, November 2006.

[28] J. J. Pan, Q. Yang, H. Chang, and D. Y. Yeung, "A manifold regularization approach to calibration reduction for sensor-network based tracking," in Proceedings of the Twenty-First National Conference on Artificial Intelligence. AAAI Press, July 2006, pp. 988-993.

[29] F. Chung, Spectral Graph Theory. American Mathematical Society, 1997.

[30] J. Shi and J. Malik, "Normalized cuts and image segmentation," IEEE Transactions on Pattern Analysis and Machine Intelligence, vol. 22, no. 8, pp. 888-905, 2000 .

[31] I. S. Dhillon, "Co-clustering documents and words using bipartite spectral graph partitioning," in Proceedings of the seventh ACM SIGKDD international conference on Knowledge discovery and data mining. ACM Press, 2001, pp. 269-274.

[32] B. Hendrickson, "Latent semantic analysis and fiedler embeddings," in Proceedings of SIAM Workshop on Text Mining. SIAM, April 2006.

[33] X. Zhu, Z. Ghahramani, and J. D. Lafferty, "Semi-supervised learning using gaussian fields and harmonic functions," in Proceedings of the Twentieth International Conference on Machine Learning. ACM Press, August 2003, pp. 912-919.

[34] N. Ravi, N. Dandekar, P. Mysore, and M. L. Littman, "Activity recognition from accelerometer data," in Proceedings of the 7th Innovative Applications of Artificial Intelligence Conference. AAAI Press, 2005, pp. 1541-1546.

[35] E. O. Brigham and R. E. Morrow, "The fast fourier transform," IEEE Spectrum, vol. 4, pp. 63-70, 1967.

[36] J. A. Ward, P. Lukowicz, and H. W. Gellersen, "Performance metrics for activity recognition," ACM Trans. Intell. Syst. Technol., vol. 2, pp. 6:1-6:23, January 2011.

[37] L. Ni, Y. Liu, Y. Lau, and A. Patil, "LANDMARC: Indoor location sensing using active RFID," in Proceedings of the First IEEE International Conference on Pervasive Computing and Communications. IEEE, March 2003, pp. 407-416.

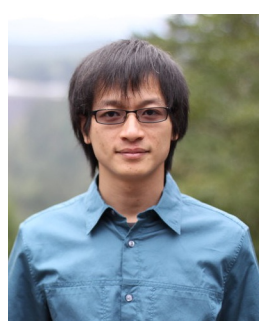

Jeffrey Junfeng Pan received the Ph.D. degree in Computer Science from the Hong Kong University of Science and Technology in 2008, and the B.S. degree in Computer Science from Sun Yat-sen University in China in 2003. He is currently a research scientist in Facebook Inc., Palo Alto, CA. He was a research engineer in Google Inc., Mountain View, CA from 2007 to 2010 . His main research interests include machine learning, data mining and their applications in ads optimization and mobile computing.

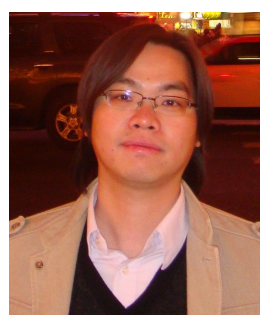

Sinno Jialin Pan received the Ph.D. degree in computer science and engineering from the Hong Kong University of Science and Technology in 2010, and the B.S. and M.S. degrees in applied mathematics from Sun Yat-sen University in China in 2003 and 2005, respectively. $\mathrm{He}$ is currently a research fellow at the Institute for Infocomm Research, Singapore. His main research interests include transfer learning, semisupervised learning and theirs applications in pervasive computing and information extraction.

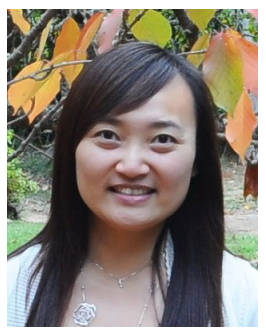

Jie Yin received the PhD degree in Computer Science from the Hong Kong University of Science and Technology in 2006, and the BE degree from Xi'an Jiaotong University, China, in 2001 . She is currently a research scientist in the Information Engineering Laboratory at CSIRO ICT Centre, Australia. Her research interests include data mining, machine learning and their applications to sensor-based activity recognition, social network analysis and Web mining.

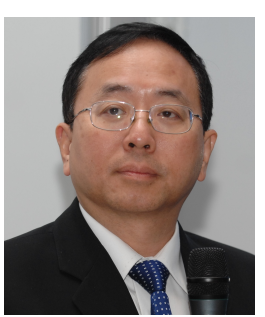

Lionel M. Ni received the Ph.D. degree in electrical and computer engineering from Purdue University, West Lafayette, IN, in 1980 . He is Chair Professor in the Computer Science and Engineering Department at HKUST. He served as the Department Head from 2002 to 2008 . He also serves as the Special Assistant to the President at HKUST, Dean of HKUST Fok Ying Tung Graduate School, and Director of HKUST China Ministry of Education/Microsoft Research Asia IT Key Lab. He was the Chief Scientist of the National Basic Research Program of China (973 Program) on Wireless Sensor Networks. He has chaired many professional conferences and has received a number of awards for authoring outstanding papers. He is a fellow of IEEE.

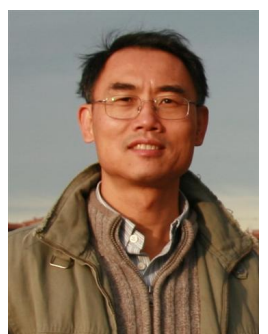

Qiang Yang is a professor in the Department of Computer Science and Engineering, Hong Kong University of Science and Technology and an IEEE Fellow. His research interests are data mining and artificial intelligence. He received his Ph.D. degree in Computer Science from the University of Maryland, College Park and B.S. degree from Peking University in Astrophysics. He was elected as a vice chair of ACM SIGART in July 2010. He is the founding Editor in Chief of the ACM Transactions on Intelligent Systems and Technology (ACM TIST). He was a PC co-chair or general co-chair for a number of international conferences, including ACM KDD'10, ACM IUI'10, etc. 